\title{
DELAYED IATROGENIC AORTIC DISSECTION FROM CORONARY BYPASS MANAGED WITH EXTRAANATOMIC BYPASS
}

\author{
Dean Pappas, MD, George L. Hines, MD, Mark Gennaro, MD, and Alan Hartman, MD, Mineola and Stony Brook, N.Y.
}

Perioperative iatrogenic dissection originating in the ascending aorta is a rare but devastating complication of cardiac operations, which occurs with an incidence of $0.16 \%$ to $0.6 \% .^{1}$ The literature recommends urgent, definitive repair of perioperative aortic dissections. ${ }^{1,2}$ However, in certain circumstances the risk of definitive repair may be greater than that of a more conservative approach. ${ }^{3}$ Multiple series quote a mortality associated with definitive repair (interposition graft or patch) of $20 \%$ to $50 \%$. ${ }^{1,2}$ In two patients we managed iatrogenic aortic dissections after cardiac operations with extraanatomic bypass of the lower extremities, and the results were excellent.

PATIENT 1. A 57-year-old man had a 1-week history of exertional chest pain and underwent a six-vessel coronary artery bypass without any noticeable complications. No significant aortic disease was noted. The patient was extubated early in the postoperative period and was in hemodynamically stable condition in the intensive care

From the Department of Thoracic and Cardiovascular Surgery, Winthrop-University Hospital, Mineola, N.Y., and the Department of Surgery, SUNY, Stony Brook, N.Y.

Received for publication Oct. 9, 1997; accepted for publication Oct. 17, 1997.

Address for reprints: George L. Hines, MD, Department of Thoracic and Cardiovascular Surgery, Winthrop-University

Hospital, 259 First St., Mineola, N.Y. 11501.

J Thorac Cardiovasc Surg 1998;115:947-9

Copyright (c) 1998 by Mosby, Inc.

$0022-5223 / 98 \$ 5.00+0 \quad \mathbf{1 2 / 5 4 / 8 7 0 4 8}$ unit. Vague bilateral leg pain developed late in the evening on the operative day, and by the morning on postoperative day (POD) 1 neither femoral pulse was palpable. He had considerable bilateral leg pain with early onset of motor and sensory loss as well as cool lower extremities. Although the patient had no other manifestations of a dissection, such as metabolic acidosis, increase in lactic acid, abdominal pain, central neurologic deficits, or evidence of renal compromise, we believed that a dissection was the most likely diagnosis. Upper extremity pressures were $140 / 70 \mathrm{~mm} \mathrm{Hg}$ bilaterally. In the operating room an intraoperative transesophageal echocardiogram confirmed our diagnosis. A large false lumen originating from a point just distal to the aortic cannulation site, down to a point below the diaphragm, was documented. We performed an axillo-bifemoral bypass, which required 3 hours of operative time. Postoperatively, perfusion of the lower extremities was restored with palpable femoral, posterior tibial, and dorsalis pedis pulses bilaterally, as well as near normal motor and sensory function. A postoperative magnetic resonance imaging scan showed no evidence of brain or spinal cord ischemic lesions. A postoperative computed tomographic scan showed a false lumen, as previously identified by intraoperative transesophageal echocardiography, and no evidence of central nervous system lesions (Fig. 1). By POD 7, the patient was walking approximately 150 feet with minor residual motor dysfunction and was subsequently discharged to a rehabilitation facility.

PATIENT 2. A 68-year-old man underwent an eventful five-vessel coronary bypass. In the postoperative period he 


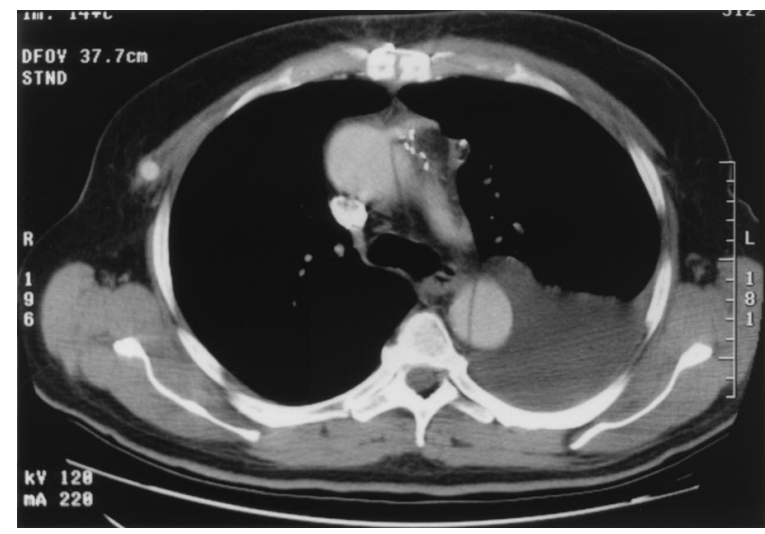

Fig. 1. Computed tomographic scan demonstrating dissection involving ascending and descending aorta.

had pneumonia in the right lower lobe for which he was treated with intravenous antibiotics. The patient was discharged on POD 9. On POD 23, he began having moderate to severe pain in the right buttock and thigh, which prompted readmission. The right femoral pulse was not palpable and signs of peripheral ischemia were evident. An emergency angiogram demonstrated occlusion of the right common iliac artery with decreased flow to the common femoral artery. There was a suggestion of an infrarenal abdominal aortic aneurysm. A computed tomographic scan demonstrated an aortic dissection arising from the aortic root. There were no other manifestations of the dissection aside from the right leg ischemia. The patient was returned to the operating room, where a transesophageal echocardiogram was performed (Fig. 2), and a left-to-right femoral-femoral bypass was created with polytetrafluoroethylene. Postoperatively, the right leg ischemia resolved with significant clinical improvement. Postopoperative magnetic resonance imaging shows no evidence of spinal cord injury, and the patient is doing well.

Discussion. Iatrogenic aortic dissections have been well described, ${ }^{4}$ with an incidence of $0.16 \%$ as shown by Still and associates ${ }^{1}$ in a review of 14,877 cases and up to $0.6 \%$ after aortic valve repair or replacement. Iatrogenic dissections may account for $3 \%$ to $5 \%$ of the total in-hospital mortality after cardiac operations. ${ }^{2}$

An intraoperative dissection usually manifests itself by bleeding from most or all of the aortic puncture sites with tense circumferential dilatation and bluish discoloration of the aorta. ${ }^{1}$ The manifestations of postoperative dissections may be as subtle as a gradual rise in the blood urea nitrogen/creatine ratio or as profound as a rapid and catastrophic hemodynamic decompensation and death. A high index of suspicion in the immediate postoperative period is critical to avoid delays in diagnosis.

Without treatment, the dissection may disrupt the recently placed grafts, coronary vessels, or aortic valve. The natural history of untreated dissections is unclear, and there are reports of dissections being diagnosed years after bypass. ${ }^{2,3}$ Those patients noted to be at risk often

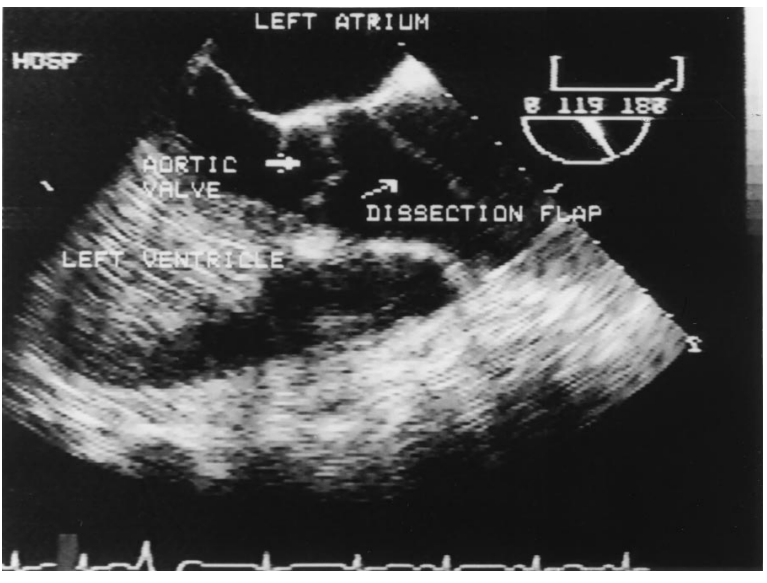

Fig. 2. Transesophageal echocardiogram demonstrating dissection flap involving ascending aorta.

have severe atherosclerotic changes of the aorta, thin dilated aortic walls, cystic medial necrosis, or collagen vascular diseases. ${ }^{1,4}$ Once the dissection is recognized, urgent definitive repair is recommended. The cases we are presenting are unique and raise some questions with regard to the management of these complications: (1) Can definitive repair be delayed or even avoided if certain clinical conditions are present (i.e., the lack of clinical evidence of aortic valve, proximal grafts, great vessel, renal, or visceral arterial involvement)? (2) What preoperative work-up is necessary before surgical intervention?

Definitive repair (interposition graft or Dacron patch) ${ }^{1}$ is clearly indicated for all dissections recognized intraoperatively or those associated with hemodynamic instability in the immediate postoperative period. Neither of our patients had evidence clinically of involvement of the great vessels, spinal cord inflow, or visceral or renal arteries. All laboratory data including blood urea nitrogen/creatine ratio, lactic acid content, and blood gases were within normal limits, and there was no evidence, clinical or laboratory, suggesting other organ system involvement. We believed that we could treat the sequela of the dissections (the lower leg ischemia) with an axillobifemoral bypass in the first case and a left-to-right femoral-femoral bypass in the second case and not subject our patients to the $50 \%$ mortality associated with postoperative repair of iatrogenic dissections. ${ }^{1}$

A second point is that intraoperative transesophageal echocardiography is an extremely accurate, relatively noninvasive tool, invaluable in the diagnosis of dissections involving the proximal aorta. Sensitivity and specificity of $99 \%$ and $98 \%$, respectively, are currently reported in the literature. Some advocate either preoperative computed tomographic scan, ${ }^{5}$ aortogram, or both before surgical intervention. We believe, however, that transesophageal echocardiography gives all the revelant characteristics of the dissection needed to plan for correction. However, if visceral or renal arterial integrity is questioned, then computed tomography or angiography may provide the necessary information. 
We thank Dr. Jason Lazar for helping with the transesophageal echocardiography.

\section{REFERENCES}

1. Still RJ, Hilgenberg AD, Akins CW, Daggett WM, Buckley MJ. Intraoperative aortic dissection. Ann Thorac Surg 1992; 53:374-80.

2. Sabri MN, Henry D, Wechsler AS, DiSciascio G, Vetrovec GW. Late complications involving the ascending aorta after cardiac surgery: recognition and management. Am Heart J 1991:121(6 Pt 1):1779-83.

3. Bopp P, Perrenoud JJ, Periat M. Dissection of ascending aorta: rare complication of aorto-coronary venous bypass surgery. Br Heart J 1981:46:571-3.

4. Nicholson WJ. Aortic root dissection complicating coronary bypass surgery. Am J Cardiol 1978;41:103-7.

5. Archer AG, Choyke PL, Zeman RK, Green CE, Zuckerman M. Aortic dissection following coronary artery bypass surgery: diagnosis by CT. Cardiovasc Intervent Radiol 1986;9:142-5. 